\title{
ENTERIC FEVER IN BASTAR TRIBAL REGION-PREVALENCE AND SENSITIVITY PATTERNS
}

\author{
Vikas Chandra Yadav¹, Vepada Ravi Kiran², Raj Sharma ${ }^{3}$
}

${ }^{1}$ Associate Professor, Department of Microbiology, LSBKM Government Medical College, Jagdalpur, Chhattisgarh. 2 Demonstrator, Department of Microbiology, LSBKM Government Medical College, Jagdalpur, Chhattisgarh. 3 Assistant Professor, Department of Pharmacology, LSBKM Government Medical College, Jagdalpur, Chhattisgarh.

ABSTRACT
OBJECTIVE
Currently, antibiotic resistance in bacterial populations is one of the greatest challenges to the effective management of
infectious diseases. The aim of this research was to study the prevalence of salmonella species and its sensitivity pattern in a
tertiary care medical college hospital in Bastar, Chhattisgarh.

\section{MATERIAL AND METHODS}

From the clinical samples cultured from Jan 2010 to June 2014. 690 cases of enteric fever were isolated and investigations were carried out for antibiotic susceptibility pattern of S. typhi and S. Paratyphi Result: Antimicrobial susceptibility pattern of S. typhi against various antibiotics tested were chloramphenicol 37\%-52\%, co-trimoxazole 22-33\%, ampicillin 28-46\%, ciprofloxacin 14-29\%, ofloxacin 80-95\%, amikacin 89\%-97\%, cefotaxime 56\%-88\%, ceftriaxone $69 \%-72 \%$, ceftazidime $86-93 \%$, and nalidixic $93-86 \%$. Sensitivity pattern of S. Paratyphi showed $100 \%$ resistance to co-trimoxazole, $40 \%$ to ciprofloxacin while they were $100 \%$ sensitive to ofloxacin and amikacin.

\section{DISCUSSION}

Emergence of MDR Salmonella is the main problem of treating the patient as a consequence of extensive use of antibiotic. Resistance to antibiotic was found with increasing frequency in our study. Hence, there should be a national policy for antibiotic usage.

\section{KEYWORDS}

MIC, MDR.

HOW TO CITE THIS ARTICLE: Yadav VC, Kiran VR, Sharma R. Enteric fever in Bastar tribal region-prevalence and sensitivity patterns. Evolution Med. Dent. Sci. 2016;5(53):3526-3530, DOI: 10.14260/jemds/2016/813

\section{INTRODUCTION}

Enteric fever is caused by S. typhi and Paratyphi types [S. Paratyphi A, S. schottmuelleri (Previously named S. typhi B), and S. hirshfeldii (previously named S. Paratyphi C)]. These species of Salmonella are restricted to humans and do not have a reservoir in animals. Therefore, spread of the infection is from person to person usually through contaminated food or water. ${ }^{1}$

The Salmonellae are the most complex of all the Enterobacteriaceae with more than 2,400 serotypes described in the current Kauffmann-White Scheme. ${ }^{2}$

In typhoid and other enteric fevers, infection begins in the small intestine, but few gastrointestinal symptoms occur. The organism enters, multiply in the mononuclear phagocytes of Peyer's patches, and then spread to the phagocytes of the liver, gallbladder, and spleen. This leads to bacteraemia, which is associated with the onset of fever and other symptoms, probably caused by endotoxin. In enteric fever, blood culture procedure is most likely to reveal the organisms during the first 2 weeks of illness. ${ }^{3}$

Financial or Other, Competing Interest: None.

Submission 17-05-2016, Peer Review 13-06-2016,

Acceptance 17-06-2016, Published 02-07-2016.

Corresponding Author:

Dr. Vikas Chandra Yadav

Associate Professor,

Department of Microbiology,

LSBKM Govt. Medical College,

Dimra Pal, Jagdalpur

Chhattisgarh.

E-mail:dr_vcyadav@yahoo.co.in

DOI: $10.14260 /$ jemds/2016/813
Typhoid fever is endemic in India where it is also a major health problem in developing countries. Reported data for the year 2005 shows 6,53,580 cases and 417 deaths in India. 4

In 2004, WHO estimated the global typhoid fever disease burden at 21 million cases annually resulting in an estimated $2,16,00$ to 60,000 deaths per year. The aetiological agents of enteric fever are S. typhi and S. Paratyphi serotypes A, B, and C. 5

There is one case of paratyphoid fever for every four cases of typhoid fever Salmonella enterica serotype Paratyphi A, which had been reported less frequently from cases of enteric fever has shown an increasing trend since 1996 in India.

New Delhi showed the rise in proportion of Salmonella Paratyphoid A from 6.5 percent in 1994 to 44.9 percent in 19984 , and there is also an increase from 11.1 percent in 2001 to 59 percent in 2003 at Calicut. At Nagpur, an annually high rate of $46.15 \%$ was reported while it was $53.33 \%$ at Sevagram. ${ }^{6}$

The present study was performed to detect the enteric fever incidence and frequency of serotypes among the patient of Pyrexia of Unknown Origin (PUO) and their antibiotic susceptibility to the more commonly used drugs like ciprofloxacin, ceftriaxone, ofloxacin, chloramphenicol etc. in patients attending a tertiary care medical college hospital in Bastar, Chhattisgarh.

\section{MATERIAL AND METHODS}

A prospective study was performed on Patients of Pyrexia suspected for enteric fever. Blood samples were collected in the Microbiology Department of a Tertiary Medical Centre at 
Jagdalpur. Blood culture of 4818 pyrexia patients who attended OPD from January 2010 to June 2014 were performed by standard method for study and proper treatment. The organisms may be recovered from the blood stream at any stage of the illness, but are most commonly found during the first 7-10 days and during relapses. ${ }^{7}$ Salmonella species were isolated by standard techniques and subjected for antimicrobial susceptibility test. 8

All culture plates showing growth of similar colonies of Salmonella species were processed for identification by biochemical reactions and confirmed by serological test with specific antisera. After identification sensitivity test was performed by Kirby-Bauer method and inference was made with the guideline of manufacturer (HiMedia).

The Widal slide agglutination test was performed according to manufacturer's (Span) instruction using reagents containing $\mathrm{O}$ and $\mathrm{H}, \mathrm{AH}$, and $\mathrm{BH}$ antigens of $\mathrm{S}$. typhi and S. Paratyphi A, Paratyphi B, respectively. Positive and negative serum controls were included at a titre of $>160$ to $\mathrm{H}$, $\mathrm{AH}, \mathrm{BH}$ antigens in a single serum specimen was taken to be indicative of typhoid fever. The results were correlated with blood culture results and interpreted accordingly.

The disc diffusion Kirby-Bauer method was used to determine susceptibility to antimicrobial agents as described by the Clinical and Laboratory Standards Institute (CLSI) guidelines of 2009. The discs of antimicrobial agents tested and reported were ampicillin (10 mg), trimethoprim- sulfamethoxazole (25/23.75), chloramphenicol (30 mg), ceftriaxone (30 mg), ciprofloxacin (5 mg), nalidixic acid (30 $\mathrm{mg}$ ), amikacin, ofloxacin, cefotaxime, and ceftazidime.

MIC of 50 isolates of S. typhi was determined by agar dilution method on the Mueller-Hinton Agar Media (HiMedia) with inocula of 105 organisms/mL followed by $24 \mathrm{hrs}$. incubation and interpreted using standard protocol. Each isolated Salmonella strain was tested against 12 dilutions of 7 antibiotics chloramphenicol, ampicillin, gentamicin, amikacin, ciprofloxacin, cefotaxime, and ceftriaxone. (Table-7).

Each new kit of antimicrobial discs were tested with E. coli ATCC 25922, Pseudomonas aeruginosa ATCC-27853 and Staphylococcus aureus ATCC-25923 [Minimum inhibitory concentration was performed by agar dilution method using Mueller-Hinton Agar (MHA) according to the CLSI guidelines]. Double dilution concentration method was used that is from $0.125 \mathrm{mg} / \mathrm{mL}$ to $512 \mathrm{mg} / \mathrm{mL}$. The MIC break point value for mcg resistant was used accordingly recommended by NCCLS. ${ }^{9}$

\section{RESULT}

From January 2010 to June 2014, 4818 pyrexia cases suspected as enteric were taken for study. Blood samples were collected from patients for blood culture and serological test. Out of the 4818 samples, a total of 690 (14.32\%) cases of pyrexia were diagnosed as enteric fever by blood culture method.

\begin{tabular}{|c|c|c|c|c|}
\hline Year & Blood Culture & Total Cases & S. Typhi & S. Paratyphi A \\
\hline 2010 & 1036 & $138(13.32 \%)$ & $122(88.40 \%)$ & $16(11.60 \%)$ \\
\hline 2011 & 0907 & $114(12.56 \%)$ & $103(90.35 \%)$ & $11(9.65 \%)$ \\
\hline 2012 & 1012 & $125(12.35 \%)$ & $111(88.8 \%)$ & $14(11.2 \%)$ \\
\hline 2013 & 1228 & $207(16.69 \%)$ & $193(92.23 \%)$ & $14(6.76 \%)$ \\
\hline 2014 (Till June) & 635 & $106(16.69 \%)$ & $101(95.23 \%)$ & $05(4.81 \%)$ \\
\hline Total Cases & $\mathbf{4 8 1 8}$ & $\mathbf{6 9 0 / 4 8 1 8}(14.32 \%)$ & $\mathbf{6 3 0}(\mathbf{9 1 . 3 0} \%)$ & $\mathbf{6 0}(\mathbf{8 . 7} \%)$ \\
\hline \multicolumn{7}{|c|}{} \\
\hline
\end{tabular}

In our study, we found common types of infecting serovar is Salmonella typhi $91.30 \%$ while only $8.70 \%$ were caused by S. Paratyphi A. A comparison of the blood culture and the Widal test reports was also done. In our Study, 87\% of cases were positive in both blood culture and Widal test. In 13\% cases, blood culture was positive while Widal test was nonreactive. 690 strains of Salmonella serotypes were isolated over a period of 4 years and 6 months that is from January 2010 to June 2014.
Out of this 690, 602 strains were also shown reactive up to a significant level by Widal serological test. In year 2010 , there were 138 cases positive out of 1036 blood culture test. In 2013, there were 207 cases out of 1228 blood culture (16.85\%). In 2014 , there were $16.69 \%$ of cases were found positive. It indicates that there is an increase in the number of cases due to Salmonella serotype infection.

\begin{tabular}{|c|c|c|c|}
\hline & Males & Females & Total \\
\hline Adults & 298 & 269 & $567(82.18 \%)$ \\
\hline Children & 72 & 51 & $123(17.82 \%)$ \\
\hline Total & $\mathbf{3 7 0}(53.62 \%)$ & $\mathbf{3 2 0}(\mathbf{4 6 . 3 8 \% )}$ & $\mathbf{6 9 0}$ \\
\hline \multicolumn{2}{|c|}{ Table 2: Incidence of Enteric Fever in Males, Females and Children (Below 12 Years) } \\
\hline
\end{tabular}

Out of these 690 cases, 370 (53.62\%) cases were males, while $320(46.38 \%)$ were females. This study show male are more prone to these diseases. Adults are also more prone to diseases as out of 690 patients of enteric fever 567 were either adults (82.18) and above 12 years of age group while 123 cases were among up to 12 years of age (17.82\%). 


\begin{tabular}{|c|c|c|c|c|c|}
\hline Antibiotics & $\mathbf{2 0 1 0}$ & $\mathbf{2 0 1 1}$ & $\mathbf{2 0 1 2}$ & $\mathbf{2 0 1 3}$ & $\mathbf{2 0 1 4}$ \\
\hline Ampicillin & $66(54 \%)$ & $60(58.25 \%)$ & $71(63.96 \%)$ & $140(72.53 \%)$ & $69(68.31 \%)$ \\
\hline Co-trimoxazole & $96(78.68 \%)$ & $70(67.96 \%)$ & $78(70.27 \%)$ & $146(75.64 \%)$ & $79(78.21 \%)$ \\
\hline Chloramphenicol & $73(59.8 \%)$ & $72(69.90 \%)$ & $61(54.95 \%)$ & $94(48.70 \%)$ & $40(39.60 \%)$ \\
\hline Ciprofloxacin & $92(75.40 \%)$ & $81(78.64 \%)$ & $79(71.17 \%)$ & $153(79.27 \%)$ & $87(86.13 \%)$ \\
\hline Amikacin & $7(05.73 \%)$ & $3(02.91 \%)$ & $12(10.81 \%)$ & $8(04.14 \%)$ & $4(3.9 \%)$ \\
\hline Ofloxacin & $24(19.67 \%)$ & $5(04.85 \%)$ & $8(07.20 \%)$ & $21(10.88 \%)$ & $14(13.86 \%)$ \\
\hline Cefotaxime & $15(12.29 \%)$ & $14(13.59 \%)$ & $20(18.01 \%)$ & $46(28.83 \%)$ & $35(34.65 \%)$ \\
\hline Ceftriaxone & $12(09.81 \%)$ & $9(08.73 \%)$ & $16(14.41 \%)$ & $29(15.02 \%)$ & $31(30.69 \%)$ \\
\hline Ceftazidime & 0 & 0 & 0 & $19(9.84 \%)$ & $5(4.9 \%)$ \\
\hline Nalidixic acid & 0 & 0 & $17(15.31 \%)$ & $34(17.6 \%)$ & $19(18.81 \%)$ \\
\hline Sensitive to all & $8(06.55 \%)$ & $6(05.82 \%)$ & $5(04.50 \%)$ & $11(5.69 \%)$ & $4(3.9 \%)$ \\
\hline
\end{tabular}

Pattern of antimicrobial susceptibility of S. typhi against various antibiotics tested was chloramphenicol: $37 \%$ to $52 \%$, co-trimoxazole: $22 \%$ to $33 \%$, ampicillin: $28 \%$ to $46 \%$, ciprofloxacin: $14 \%$ to $29 \%$, ofloxacin: $80 \%$ to $95 \%$, amikacin: $89 \%$ to $97 \%$, cefotaxime: $56 \%$ to $88 \%$, ceftriaxone: $69 \%$ to $92 \%$, ceftazidime: $86 \%$ to $93 \%$, and nalidixic: $93 \%$ to $86 \%$.
In the present study, ampicillin, co-trimoxazole, and ciprofloxacin showed a downward trend. Chloramphenicol showed an upward trend in the sensitivity pattern. All the newer drugs e.g. amikacin, ofloxacin, cephalosporin (Ceftazidime), ceftriaxone, nalidixic acid were more effective. However, some cases of antibiotic resistance in isolates ranging from $5 \%$ to $30 \%$ have been encountered recently in our study.

\begin{tabular}{|c|c|c|c|c|c|}
\hline Antibiotics & $\mathbf{2 0 1 0}$ & $\mathbf{2 0 1 1}$ & $\mathbf{2 0 1 2}$ & $\mathbf{2 0 1 3}$ & $\mathbf{2 0 1 4}$ \\
\hline Ampicillin & $14(87.5 \%)$ & $10(90 \%)$ & $12(85.71 \%)$ & $10(71.42 \%)$ & $3(60 \%)$ \\
\hline Co-trimoxazole & $16(100 \%)$ & $8(72.7 \%)$ & $11(78.57 \%)$ & $9(64.28 \%)$ & $4(80 \%)$ \\
\hline Chloramphenicol & $10(62.5 \%)$ & $8(72.7 \%)$ & $7(50 \%)$ & $8(57.14 \%)$ & $3(60 \%)$ \\
\hline Ciprofloxacin & $6(37.5 \%)$ & $5(45.45 \%)$ & $8(57.14 \%)$ & $9(64.28 \%)$ & $4(80 \%)$ \\
\hline Amikacin & $0(0 \%)$ & $0(0 \%)$ & $0(0 \%)$ & $1(7.4 \%)$ & $0(0 \%)$ \\
\hline Ofloxacin & $0(0 \%)$ & $2(18.18 \%)$ & $3(21.42 \%)$ & $4(28.57 \%)$ & $1(20 \%)$ \\
\hline Cefotaxime & $2(12.5 \%)$ & $3(27.27 \%)$ & $6(42.85 \%)$ & $7(50 \%)$ & $2(40 \%)$ \\
\hline Ceftriaxone & $0(0 \%)$ & $2(18.18 \%)$ & $3(21.42 \%)$ & $2(14.28 \%)$ & $1(20 \%)$ \\
\hline Ceftazidime & $0(0 \%)$ & $0(0 \%)$ & $2(14.28 \%)$ & $1(7.14 \%)$ & $1(20 \%)$ \\
\hline Nalidixic acid & $0(0 \%)$ & $0(0 \%)$ & $1(7.4 \%)$ & $2(14.28 \%)$ & $0(0 \%)$ \\
\hline Sensitive to all & $0(0 \%)$ & $0(0 \%)$ & $2(14.28 \%)$ & $4(28.57 \%)$ & $0(0 \%)$ \\
\hline \multicolumn{7}{|l|}{ Table 4: S. Paratyphi showing Resistance Against Chemotherapeutic Agents } \\
\hline
\end{tabular}

The antimicrobial sensitivity pattern of S. Paratyphi against the various antibiotics is shown in Table 4, from January 2010 to June 2014. In our study, S. Paratyphi A 100\% resistance to co-trimoxazole was seen in 2010 and showed a decreasing trend to $64 \%$ in 2013 , but increased again and in 2014 to $8 \%$.

Ampicillin resistance was high in 2010-2011, i.e. $87 \%$ to $90 \%$, which decreased from 2012 onwards to $60 \%$ in 2014 . Ciprofloxacin was $63 \%$ sensitive in 2010, which showed a decreasing pattern to $40 \%$ in 2010 . Sensitivity pattern of chloramphenicol was increasing from 38\% in 2010 to $50 \%$ in 2012 and again from $43 \%$ to $40 \%$ in $2013-2014$. Ofloxacin and amikacin were $100 \%$ sensitive in 2010 . Amikacin is still $90 \%$ sensitive in 2013 while ofloxacin is $80 \%$ sensitive against the S. Paratyphi A.

There is a decreasing pattern of sensitivity against third generation group; cephalosporin ceftriaxone, cefotaxime, or ceftazidime ranges from $88 \%$ to $100 \%$ sensitive in 2010 to 60 to $93 \%$ in 2013.

\begin{tabular}{|c|c|c|c|}
\hline Year (Cases) & MDR 4 ACCOTCF & MDR3 ACCOT & MDR2 \\
\hline $2010(122)$ & $5(4.09 \%)$ & $22(18.03 \%)$ & $72(59 \%)$ \\
\hline $2011(103)$ & $3(02.91 \%)$ & $14(13.59 \%)$ & $66(64.07 \%)$ \\
\hline $2012(111)$ & $3(02.70 \%)$ & $12(10.81 \%)$ & $61(54.95 \%)$ \\
\hline $2013(193)$ & $7(03.62 \%)$ & $44(22.79 \%)$ & $132(68.39 \%)$ \\
\hline $2014(101)$ & $4(03.96 \%)$ & $16(15.84 \%)$ & $65(64.35 \%)$ \\
\hline \multicolumn{4}{|c|}{ Table 5: S. typhi showing MDR } \\
\hline
\end{tabular}




\begin{tabular}{|c|c|c|c|}
\hline Year (Cases) & MDR4 ACCOTCF & MDR3 ACCOT & MDR2 \\
\hline $2010(16)$ & $0(0 \%)$ & $1(6.25 \%)$ & $2(12.5 \%)$ \\
\hline $2011(11)$ & $0(0 \%)$ & $0(0 \%)$ & $2(18.18 \%)$ \\
\hline $2012(14)$ & $1(7.14 \%)$ & $12(14.28 \%)$ & $2(14.28 \%)$ \\
\hline $2013(14)$ & $1(7.14 \%)$ & $1(7.14 \%)$ & $2(14.28 \%)$ \\
\hline $2014(05)$ & $0(0 \%)$ & $0(0 \%)$ & $0(0 \%)$ \\
\hline \multicolumn{2}{|c|}{ Table 6: S. Paratyphi showing MDR } \\
\hline
\end{tabular}

Multidrug resistance i.e. resistance to three or more chemotherapeutic drug in S. typhi and S. Paratyphi A is as shown in Table 5 and Table 6 respectively.
MDR in S. typhi from 2010 to 2014 was found in 130 cases out of 630 isolated (21\%). It showed an upward trend from 2012. MDR in S. Paratyphi was $10 \%$ of isolated strains i.e. 6 out of 60 cases in our study.

\begin{tabular}{|c|c|c|c|c|c|c|c|}
\hline$\underset{\mathrm{mg} / \mathrm{mL}}{\operatorname{Drug}}$ & $\begin{array}{l}\text { Chlor } \\
(>32)\end{array}$ & $\begin{array}{l}\text { Ampi } \\
(>32)\end{array}$ & $\begin{array}{c}\text { Genta } \\
(>8)\end{array}$ & $\begin{array}{c}\text { Amika } \\
(>32)\end{array}$ & $\begin{array}{c}\text { Cipro } \\
(>4)\end{array}$ & $\begin{array}{c}\text { Cefotax } \\
(>64)\end{array}$ & $\underset{(>64)}{\text { Ceftriax }}$ \\
\hline$>128$ & 8 & 00 & ** & ** & 00 & $* *$ & $* *$ \\
\hline$>64$ & 8 & 44 & 00 & 00 & 00 & 00 & 00 \\
\hline$>32$ & 06 & 04 & 00 & 04 & 04 & 02 & 00 \\
\hline$>16$ & 02 & 02 & 00 & 20 & 20 & 04 & 00 \\
\hline$>08$ & 12 & 00 & 00 & 00 & 00 & 12 & 08 \\
\hline$>04$ & 04 & 00 & 12 & 06 & 08 & 08 & 16 \\
\hline$>02$ & 02 & 00 & 04 & 04 & 08 & 04 & 4 \\
\hline$>01$ & 02 & 00 & 00 & 16 & 02 & 02 & 2 \\
\hline$>0.5$ & 06 & 00 & 04 & 14 & 04 & 08 & 2 \\
\hline$>0.25$ & 00 & 00 & 20 & 00 & 00 & 16 & 12 \\
\hline$>0.125$ & 00 & 00 & 08 & 10 & 02 & 04 & 04 \\
\hline$>0.06$ & 00 & 00 & 02 & 00 & 02 & 00 & 02 \\
\hline$>0.03$ & 00 & 00 & 00 & 00 & 00 & 00 & 00 \\
\hline \multicolumn{8}{|c|}{ Table: 7 Result of MIC Studies } \\
\hline
\end{tabular}

Numbers in each row indicate the number of strains with that MIC value against each antimicrobial agent. Ampiampicillin, Chlor-chloramphenicol, Genta-gentamicin, Cipro-ciprofloxacin, cefotax-cefotaxime, ceftria-ceftriaxone, ${ }^{* * d i l u t i o n}$ not made of that particular antibiotic.

The MIC of 50 isolates of S. typhi was determined. Only $40 \%$ (20) of these Salmonella were found to be sensitive to ciprofloxacin (Break point MIC being $4 \mathrm{mg} / \mathrm{mL}$ ). 100\% of strains were sensitive to amikacin, break point $(>32)$ as $70 \%$ of the strains were sensitive to chloramphenicol break point MIC (32 mg/mL) S. typhi strains were found to be $100 \%$ sensitive to cefotaxime, $(32 \mathrm{mg} / \mathrm{mL})$, ceftriaxone $(08 \mathrm{mg} / \mathrm{mL}$ $0.006 \mathrm{mg} / \mathrm{mL})$, and amikacin $(04 \mathrm{mg} / \mathrm{mL}-0.125 \mathrm{mg} / \mathrm{mL})$. More than $90 \%$ of the strains were resistant to ampicillin (MIC $64 \mathrm{mg} / \mathrm{mL}$ ).

\section{DISCUSSION}

Typhoid fever is endemic in India and reports for the year 1995 shows 3,29,499 cases and 672 deaths 4 . The primary sources of infection are faeces, urine of cases and carriers, the secondary sources are contaminated food, water, finger, and flies. Typhoid fevers are observed all through the year. The peak incidence is reported during June-September, this period coincides with the rainy season, when generally safe drinking water is not available in many areas. Where there is no improvement in sanitation and a large number of carriers are present in the society. The situation is compounded by the emerging resistance to antibiotics that were effective earlier.

Numerous outbreaks due to MDR Salmonella are reported from different parts of the world in general and India in particular. Our data also confirms that resistance to antimicrobial agents and in particular to traditional agents.

(Ampicillin, chloramphenicol, and co-trimoxazole) has continued to increase at an alarming pace. Antimicrobial resistance of S. typhi was rare prior to the 1980s. Chloramphenicol was the treatment of choice, but in the 1980s resistance to chloramphenicol and alternative agents began to emerge in India as in other countries where S. typhi was endemic. Thus, ciprofloxacin became the most effective treatment for typhoid. ${ }^{10}$

Strains of S. typhi showing resistance to chloramphenicol, ampicillin, and TMP/SMX were reported in South America, Africa, and Saudi Arabia and in other studies from India while at the same time in Southern Vietnam similar results including multidrug resistance became established by early 1990. Wide variation in the sensitivity pattern of various strains circulating in different geographic regions in India makes it necessary to assess the sensitivity of infecting organism/bacilli to chemotherapeutic agent before introduction of therapy. This is also important to make data from specific zones for epidemiological analysis. In Mumbai, India, nalidixic acid resistance in S. typhi increased to 79\% in 2003-2005.11 Varsha Gupta, Jaspal Kaur, and Jagdish Chander reported resistance to nalidixic acid (92.5\%) and chloramphenicol sensitivity (90\%) re-emerged, and sensitivity to ampicillin dropped to $72.5 \%$ of isolates. Only one isolate was reported as MDR. ${ }^{12}$ As a consequence of 
extensive use of fluoroquinolones resistance is being reported with increasing frequency all over the world and a correlation between resistance to nalidixic acid and reduced susceptibility to ciprofloxacin and other fluoroquinolones has been reported.13,14,15,16,17

Another study in the US from 1985-1989 reported MDRST $0.6 \%$ and from 1990-1994 MDRST rose to $12 \%$ of all strains. ${ }^{18}$ Marta-Louise Ackers, Nancy D. Puhr et al, in another study in the USA found $17 \%$ isolates were MDRST ${ }^{19}$ shows S. typhi resistance to nalidixic acid increased from $6.8 \%$ in $1996-97$ to $23.2 \%$ in 2000 , indicating a global increase of resistance to nalidixic acid. Nalidixic acid resistant ciprofloxacin susceptible S. typhi caused an outbreak in Tajikistan involving more than 6000 patients in $1997 .{ }^{20} \mathrm{~A}$ similar increase to such resistant strains also appear in the US in which most of these strains also appear in the US in which most of these strains were obtained by people travelling aboard, indication the global spread of these strains.

In 2008, there were reports of high level ciprofloxacinresistant Salmonella enteric from many centres in India, ${ }^{21}$ 22 including our study, prevalence was found to be $14.32 \%$. By in vitro antibiotic sensitivity testing, our data also confirm that resistance to antimicrobial agents (Nalidixic acid, ciprofloxacin, trimethoprim, and sulfamethoxazole) has continued to increase at an alarming pace. The emergence of resistance is due to improper use of antibiotics use in sublethal doses and in improper course.

The above study shows that prevalence of enteric fever was found to be high and most commonly observed in adult age group $82 \%$. Enteric fever is a common health problem in developing countries like India, Bangladesh, Sri Lanka, Nepal, and others where water supplies and sanitation are substandard. Large number of bacterial organisms and Salmonella species is excreted in urine and faeces specimen. During the diseases, an infection is caused by the entry of the Salmonella species through contaminated food or water or directly from faeces and contaminated fingers. Prevention of enteric fever is mainly by personal and domestic hygiene.

We conclude our study with the following recommendations for antibiotic usage to prevent the emergence of MDR strain,

- There should be a national antibiotic usage policy.

- Antibiotic drug should be available to pubic only on prescription of a registered medical personnel.

\section{REFERENCES}

1. Mims C, Dockrell HM, Goering RV, et al. Derek Wakelin \& Mark Zuckerman, medical microbiology. $3^{\text {rd }}$ ed. by Elsevier, Spain Chapter 22, 2004:301.

2. Winn W, Allen S, Janda W, et al. Koneman's Color Atlas and Textbook of Diagnostic Microbiology. $6^{\text {th }}$ ed. U.S.A Chapter 6, 2006:251.

3. Levinson W, Jawetz E. (Lange), Medical microbiology and immunology. $6^{\text {th }}$ ed. Singapore Chapter $18,2000: 115-6$.

4. Govt. of India, Health information of India 2005, Ministry of Health \& Welfare, New Delhi 2006.

5. WHO. Weekly epidemiological record no 6, 2008.
6. Tankhiwale SS, Agrawal G, Jalgaonkar SV. A preliminary report on current antibiogram of Salmonella enteric serotype typhi in Nagpur. IJMM 2003;21(4):292.

7. Wood DG, Slack R, Peutherer J, et al. Medical Microbiology. 16th ed. New Delhi Chapter 24, 2006:255.

8. Miles RS, Amyes SGB, Colle JG, et al. Mackie \& McCartney practical medical microbiology. India Chapter no 8, 2006:151-78,

9. National committee for clinical laboratory standards. performance standards for antimicrobial susceptibility testing. $6^{\text {th }}$ ed. Approved standards M2-A6, Wayne, Pennsylvania 1997.

10. Parry CM, Hein TT, Dougan G, et al. Typhoid fever. N Eng J med 2002;347(22):1770-82.

11. Jog S, Soman R, Singhal T, et al. Enteric fever in Mumbaiclinical profile, sensitivity patterns and response to antimicrobials. Journal of the association of physicians of India 2008; 56:237-2008.

12. Gupta V, Kaur J, Chander J. An increase in enteric fever cases due to Salmonella Paratyphi a in and around Chandigarh. Indian J Med Res 2009;129:95-8.

13. Chandel DS, Chaudhry R, Dhawan B, et al. Drug-resistant Salmonella enteric serotype Paratyphi a in India. Emerg infect Dis 2000;6(4):420-1.

14. Hakanen A, Kotilainen P, Jolava J, et al. Detection of decreased fluoroquinolone susceptibility and validation of nalidixic acid screening test. J Clin Microbiol 1999;37(11):3572-7.

15. Hakanen A, Kotilainen P, Huovinen P, et al. Reduced fluoroquinolone susceptibility in salmonella enteric serotypes in travellers returning from southeast Asia. Emerg Infect Dis 2001;7(6):996-1003.

16. Threlfall EJ, Ward LR. Decreased susceptibility to ciprofloxacin in salmonella enterica serotype typhi, united kingdom. Emerg Infect Dis 2001;7(3):448-50.

17. Wain J, Hoa NT, Chinh NT, et al. Quinolone-resistant salmonella typhi in viet nam: molecular basis of resistance and clinical response to treatment. Clin Infect Dis 1997;25(6):1404-10.

18. Mermin JH, Townes JM, Gerber M, et al. Typhoid fever in the United States, 1985-1994: changing risks of international travel and increasing antimicrobial resistance. Arch Intern Med 1998;158(6):633-8.

19. Marta-Louise A, Puhr ND, Tauxe RV, et al. Laboratorybased surveillance of salmonella serotype typhi infections in the United States. Journal of American Medical Association 2000;283(20):2668-73.

20. Murdoch DA, Banatvaia N, Bone A, et al. Epidemic ciprofloxacin resistant salmonella typhi in Tajikistan. Lancet 1998;351(9099):339.

21. Dutta S, Sur D, Manna B, et al. Emergence of highly fluoroquinolone-resistant Salmonella enteric serovar typhi in a community-based fever surveillance from Kolkata, India. Int J antimicrob agents 2008;31(4):387-9.

22. Harish BN, Menezes GA, Sarangapani K, et al. A case report of the literature: ciprofloxacin resistant salmonella enteric serovar typhi in India. Journal of infection diseases in Developing Countries 2008;2(4):324-7. 